\title{
The Eventuality of Equating Energy and Mass - Laws and Theories
}

\author{
Hamdoon A. Khan \\ Independent researcher, Sri Lanka \\ dr.hamdhoonkhan@gmail.com
}

Received August 2021

Received in revised: August 2021

Published: Sept 2021

\begin{abstract}
Einstein's famous equation, $E=m c^{2}$, revolutionized the theory of physics and introduced new perspectives to the study of energy and mass. However, a close consideration of its principles raises essential concerns on the equitability of mass and energy as well as other phenomena like the speed of light. The unavoidable scientific claim of this paper is that the total energy of matter depends on its internal and external energies, which are accounted for by kinetic and potential energies. In the current work, thought experiments reveal important additions to this idea regarding the apparent effects of external energy on the nature of matter and particles. This paper employs detailed thought experiments and theoretical discussions to identify and address several notable inconsistencies related to the energy and mass equation based on previous works in physics. The relative external energy of an object will be influenced by the position of the observer. The outcomes of the experiments presented herein also provide key insights into the constancy of the internal energy of all matter and particles. Generally, this paper provides an important basis for analyzing the theory underlying the physics of energy and mass, addressing questionable ideas that are common but poorly substantiated and providing a new understanding of the nature of mass and energy that lays the foundations for further research in this area by projecting the difference between them.
\end{abstract}

Keywords: Energy-Mass-Types of energy-Laws of energy-light-Internal energy.

C2021 The Authors. Published by Fundamental Journals. This is an open-access article under the CC BY-NC https://creativecommons.org/licenses/by-nc/4.0/

\section{INTRODUCTION}

The invariance of the speed of light and the equivalence of energy and mass are the greatest potent fundamental scientific concepts known to man and the most significant discoveries of the 19th and 20th centuries, a period that has introduced many amazing scientific discoveries to the world. It was during this time that the world's most famous equation, $E=m c^{2}$, was revealed. However, I believe that the immense popularity of this equation forbids us from thinking about the veracity of its argument over time. When we subject this equation to scrutiny, the first question we will encounter is whether our belief in the equivalence of energy and mass as declared by this famous equation is possible or not.

Moreover, we are certain that the speed of light in a vacuum is constant and that it can never be affected or changed by the relativity of observers. Nevertheless, have we ever asked 
ourselves since 1905 again why it is constant? Are there any ways in which that may change? Is constancy a particularity of the speed of light itself, or is there anything else that shares this quality of constancy?

We must answer these questions in every way, or risk being misled into believing in fictions and falsehoods; or, rather, further so. The theory I reveal in this paper has the potential to further our understanding of physics and aid future discoveries. Therefore, this research paper is intended to answer interesting and important questions like those raised above. Even though decades of research have been conducted on the equivalence of energy and mass since the days of Einstein, none of them have approached the keynote from a centralized point of view. Instead, Einstein originated the claim of equivalence, and others simply endorsed it. Therefore, the call of science to approach this matter from a wider and different perspective has not yet been answered. Thus, I have filled this gap and made the path clear for others by answering the call of science through this paper. This has been done by carrying out several thought experiments and logical arguments as I believe them to be strong enough to provide a clearer picture of the darkest parts of this topic.

Though the foundations on which the equation $E=m c^{2}$ is built, were completely ruined with enough scientific and philosophical evidence by the paper entitled 'The ultimate sophistication of special relativity'(Khan, 2021), it worth considering this equation here in order to bring an intelligible understanding of our universe and to enlighten our conception of energy from a wider perspective.

One must understand the sixth law of energy clearly to get a better picture of the first law. Also, must refer to my discussion about light to understand the second and third laws of energy more efficiently. I thoroughly believe that this research paper will give a clearer image of the notion of energy and mass, as well as a new approach to the speed of light.

\section{ENERGY \\ Law 01: The total energy of matter or a particle relies on its internal and external energies.}

Explanation: The energy of all matter and particles can manifest in only three types: internal, external, and total.

Internal energy is the energy a system (matter or particle) contains from its relative attributes, its motion, and the interaction of its parts. This means the energy presents in a system from its own parts such as microscopic kinetic energy, molecular attractive forces, and so forth.

External energy is the energy a matter or particle receives from the outside. In other words, the energy comes from a matter or particle and acts on another system (matter or particle) or against it, is known as external energy e.g. boiling water with fire, the potential energy acts on a suspended ball, the friction acts against the rotation of a car's tires and so on.

The total energy is the entire amount of energy present in a matter or a particle at a given time.

To bring this closer to our understanding, we must discuss two different thought experiments.

Thought experiment 01: $100 \mathrm{~g}$ of clay, stone, and iron were prepared in a spherical shape, and three different mirrors with the same quality, shape, size, and mass were placed at a distance of $10 \mathrm{~m}$. The three items were then thrown into the mirrors to collide at the speed of $27 \mathrm{~m} / \mathrm{s}$. Would any two or more of these mirrors have been experienced to the same amount of energy?

Of course not! We never get the same results at all.

Thought experiment 02: $100 \mathrm{~g}$ of paper was prepared, and a tin was placed at a distance of $10 \mathrm{~m}$. Then the paper was thrown into the tin to collide at $27 \mathrm{~m} / \mathrm{s}$ speed. Then, it has been repeated the same activity after the paper was compressed well or to the max. Now, what do you think of these two situations? Would the sound frequency of both events have been equal or not?

Exactly, they would not be equal. With these two experiments, we can prove two arguments.

1. There is something we failed to include in the equation $E=$ $m c^{2}$, i.e., the internal energy. However, the results of our experiments are something we would not get if the equation $E=m c^{2}$ is correct.

2. The total energy of matter or a particle is influenced by both internal and external energies, so we would get different outcomes for the total energy for the above experiments, even though the mass and speed of all the items were equal. Since it is not possible to get the exact amount of total energy present in any matter or particle using $E=m c^{2}$ as it claims the energy of a $100 \mathrm{~g}$ paper and plutonium to be equal, we have to propose an accurate equation for it.

$$
\text { Total Energy = Internal Energy + External Energy }
$$

For this hypothesis, the internal energy will be derived from the sum of all the molecular kinetic energy (translational motion, rotational motion, and vibrational motion) + the sum of the molecular potential energy (intermolecular bonding). Conversely, external energy will be the total kinetic energy influencing the matter or particle other than its molecular kinetic energy + the sum of the total potential energy influencing the matter or particle other than its molecular potential energy. So, the combined equation will have the terms

Kinetic energy $_{(\mathrm{e})}+$ Kinetic energy $\left._{(\mathrm{i})}\right)+\left(\right.$ Potential energy $_{(\mathrm{e})}+$ Potential energy $(\mathrm{i})$, which is the same as,

\section{$\left(\right.$ Kinetic Energy $_{(\mathrm{i})}+$ Potential Energy $\left._{(\mathrm{i})}\right)+$} (Kinetic Energy $_{(\mathrm{e})}+$ Potential Energy $\left._{(\mathrm{e})}\right)$

As you can see, the equation has two different terms (external energy and internal energy) joined by an addition sign. For each term, there is a value for kinetic energy and one for potential energy. However, the potential energy we refer to for the internal energy term is different from that for the external energy term. Since we have to use the same basic equations and symbols for the kinetic and potential energies in both the internal and external energy terms, we have to introduce new symbols subscripted ' $i$ ' for internal energy, and ' $e$ ' for external energy, as below:

$$
\begin{array}{llll}
i & 1 / 2 \mathrm{mv}^{2}{ }_{(\mathrm{i})} & \text { iii } & \mathrm{V}_{(\mathrm{i})} \\
\text { ii } & 1 / 2 \mathrm{mv}^{2}{ }_{(\mathrm{e})} & \text { iv } & \mathrm{V}_{(\mathrm{e})}
\end{array}
$$


Using these terms, we get

$$
\text { Total Energy }=\left(1 / 2 \mathrm{mv}^{2}{ }_{(\mathrm{i})}+\mathrm{V}_{(\mathrm{i})}\right)+\left(1 / 2 \mathrm{mv}_{(\mathrm{e})}^{2}+\mathrm{V}_{(\mathrm{e})}\right)
$$

where $M$ represents the mass, $v$ represents the velocity, $V_{(i)}$ represents the intermolecular bonding/ force, and $V_{(\boldsymbol{e})}$ is external potential energy. This means

$$
E=U+T
$$

where $\mathrm{E}$ represents the total energy of a matter or particle, $U$ represents its internal energy, and $T$ represents the external energy. Here, I have used the letter $T$ from the Arabic word 'Taqa', which means energy, to represent external energy. Unexpectedly, this is one of two equations that could help us in nuclear research to calculate the total energy of a system. If the external energy of a matter or particle is only in the form of kinetic energy, we can use the equation below to calculate its total energy:

$$
E=U+\frac{1}{2} m v^{2}
$$

where E represents the total energy, $U$ represents the internal energy, $\mathrm{m}$ stands for mass, and $\mathrm{v}$ for velocity.

Hypothetically, if $E=m c^{2}$ is a precise equation, we would have "observed" the same results in all of the experiments above. Furthermore, we would have succeeded in nuclear research using any element of the periodic table. However, the dissimilarity of the thought experiment results and the impossibility of succeeding in the nuclear researches using all the elements of the periodic table give us concrete evidence that there is something we have failed to consider in the equation $E=m c^{2}$, and that is internal energy. Because the dissimilar results of the thought experiments are due to differences in internal energy only.

Some may provide a few rare examples of some situations where the energy is converted into a mass or vice versa as per their belief in the equation $E=m c^{2}$. For such arguments, there is nothing to say except to request that they recheck their understanding of the equation $E=m c^{2}$ because, clearly, the equation does not explain certain specific situations or types of matter particularly. Rather, it talks about equating energy and mass in general. Thus, if we could observe examples of the interconversion of all types of energy and mass in our daily lives, their argument and the above equation would be correct. For example, take the formula for calculating profit percentages, which is revenue minus cost of services or goods sold divided by the cost of services or goods sold and multiplied by a hundred. This formula is the same for all types of businesses all over the world for everyone. Nevertheless, if someone said that this is a standard formula, but that it only applies to the profit percentage of Russian-made watches, it would never be accepted as a formula at all, and we would have to question the understanding and knowledge of those who accept it is a formula.

It is not possible to observe examples of $E=m c^{2}$ other than some hypothetical examples with no relationship to practical reality. Therefore, such examples cannot be evidence of the above equation. Rather, they serve to illustrate different concepts than those under discussion here. If the energy and mass are equivalent, the possibility must exist to obtain from both the matter and particle the pure state of each, i.e., mass without energy and vice versa. If we say that liquid water and ice are equivalent but different manifestations of the same thing, it is a demonstrably true statement. because we can observe $100 \%$ liquid water without ice and vice versa. However, this is not possible with energy and mass because matter and particles possess energy in all situations. So, hypothetically if we convert matter or a particle into energy completely, the total energy of it will be as below:

The energy present in the matter or particle before the transformation plus the energy converted from the mass via equivalence process is not equal to the mass at all, but it is more than the mass, as is clear here. This point alone is good enough to prove the incorrectness of the claim of $E=m c^{2}$ for the equivalence of energy and mass.

\section{Law 02: External energy cannot make any changes in internal energy directly without changing the matter or particle. \\ Explanation}

Thought experiment: $50 \mathrm{~g}$ of iron was heated consistently up to $900{ }^{\circ} \mathrm{C}$ and left at $0{ }^{\circ} \mathrm{C}$ for $20 \mathrm{~min}$. This activity was repeated two times. Each time, the higher and lower temperatures were selected randomly. Through this experiment, do you think that we can observe any changes in its internal energy? Undoubtedly, we can. But what is the direct cause of these changes? Is that external energy?

To get the correct answer to this question, I have to explain the above experiment from a different point of view. That is to say, when that specific iron is heated, its attributes also begin to change. On the other hand, I can say that there are no solids that will melt in an instant or vice versa. There are thousands of stages before achieving melting point as the heat changes little by little. In each of these stages, the attributes of the solid will also be changed.

To make it clearer to understand, let us repeat the same experiment from a different viewpoint. If matter or a particle has been subjected to be changed by the external energy, its attributes will be changed accordingly. However, its internal energy will be changed only according to the attributes of the matter or particle at a given point in time. This means external energy cannot make any changes in the internal energy of any matter or particle directly. So, if no change has been done to the matter or particle by any external energy, its internal energy will never be affected by that external energy at all. Also, we can give a $1 \mathrm{~m}^{3}$ brick broken using external energy as further proof of this argument. As we all know, the internal energy of that brick would not be the same before and after being broken. Nevertheless, if you say that external energy is the cause of the change in its internal energy, you are mistaken. However, the changes in the attributes of the brick caused the change in its internal energy, and the changes in the attributes of the brick were caused by external energy.

Here, one may ask, "if external energy can change the internal energy by changing the attributes of matter or a particle, why don't we simply accept the fact that the external energy can change the internal energy?" 
This is because, hypothetically, if we agree that the internal energy will be changed by the external energy, there should always be a change in internal energy whenever external energy exists. But fortunately, this is belied by reality and practical consideration. So, that kind of situation can be excluded using my second law of energy. Based on the fact that when there is no change in the attributes of matter or particles, the change in internal energy is constant.

$$
\Delta U \times E=\Delta A_{s} \times E \quad \Delta U=\Delta A_{S}
$$

where $\Delta U$ stands for changes in internal energy, $\Delta A_{s}$ stands for changes in the attributes of the system, and $E$ represents the external energy. The exact point being proved with the equations to calculate the change in internal energy.

$$
\Delta \mathrm{U}=\int_{\mathrm{T}_{1}}^{\mathrm{T}_{2}} \mathrm{C}_{\mathrm{V}} \mathrm{dT}+\int_{\mathrm{V}_{1}}^{\mathrm{V}_{2}}\left[\mathrm{~T}\left(\frac{\partial \mathrm{p}}{\partial \mathrm{T}}\right)_{\mathrm{V}}-\mathrm{p}\right] \mathrm{dV}
$$

As the attribute of a system could be defined as its temperature, volume, and pressure ( $T, V$, and $p$ ) if the change in them is zero, the changes in the internal energy also will be zero. For this argument, I can use a real-life practical example. However, we will talk about this when we are done with the thought experiments to explain the reasons behind the constancy of the speed of light for the sake of a more logical progression.

Law 03: External and total energies are relative, unlike internal energy, which cannot be relatively observed.

Law 04: There is no relativity within the same frame of reference.

Explanation: It is not meant by relativity here what usually was understood in the past century from the word. As it is proved by Hamdoon's all four paradoxes of special relativity, there is no place for the notion of relativity within the real universe. Nevertheless, there will be the relativity of motion when the reference frames of the observers are different. The space and time coordinates, motion by the external energy, and displacement in the space are the only forms of relativity worth to be considered in physical science. Though the motion is relative as said above, all three types of energy will not be relative. When an event takes place somewhere in the space, we can record them using different coordinates as below.

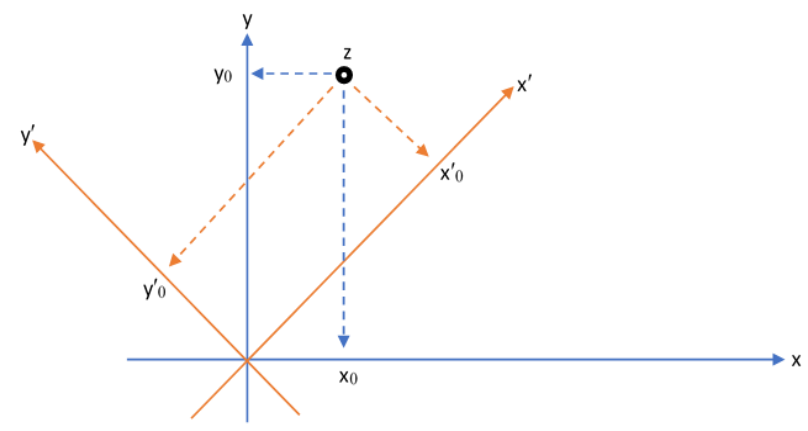

Figure 1
As shown in the picture, the point $\mathrm{z}$ could be recorded as

$$
z=\left(x_{0}, y_{0}\right) \text { or }=\left(x_{0}^{\prime}, y_{0}^{\prime}\right)
$$

The time of that event could be mentioned for an example (GMT+5:30) 00:00 Hrs, and (GMT+3) 21:30 Hrs. As it is already clear to us from the discussion about my first law of energy that the energy contained by matter or a particle from the relative attributes of the system, motion, and interaction of its parts is known as internal energy. Since the internal energy is owned by a system, it cannot exist independently. Therefore, changes in the internal energy are relative to the attributes of that matter or particle. However, the relativity of the internal energy cannot be found in all situations like external and total energies because this relativity will be bound with the changes in the attributes of matter or a particle, but will not be relative to any observers. Furthermore, It is proved by the discussion about the second law of energy and equation (7) that there will be no changes in the internal energy of a system as long as its attribute is unchanged. The research article entitled 'The ultimate sophistication of special relativity' clearly shows that the motion cannot bring any changes to the attribute on a system. Therefore, the internal energy remains constant except for a unique situation that will be discussed in the fifth law of energy. The relativity of motion and the displacement in space take place under said conditions only with the external energy.

Relativity of motion: There are two observers; one at the platform and the second on a moving train with velocity $v$. The observer on the train threw a ball in the direction of his motion in velocity $S$

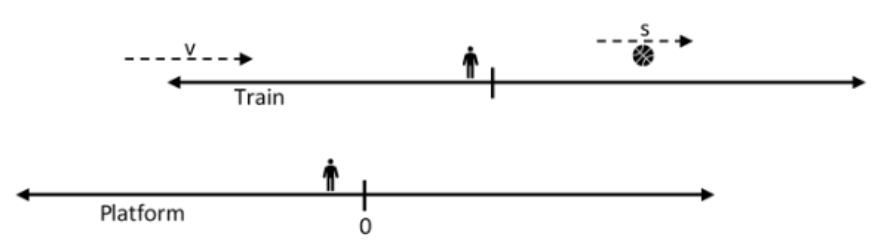

Figure 2

The velocity of the ball will be $s$ for train observer and $v+s$ dor platform observer.

Relativity of displacement in space: Let's consider the same example of the relativity of motion. But now we are going to use that to find the location on that ball from the perspective of both the observers when it reaches point a in the $x^{\prime}$.

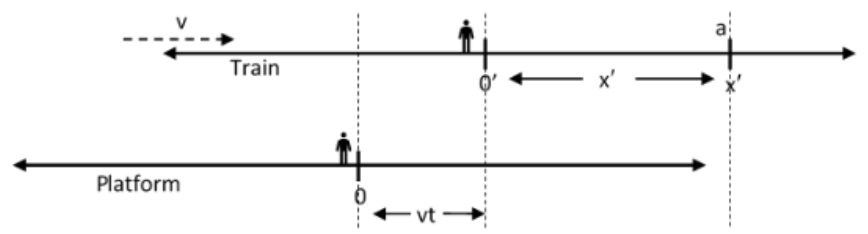

Figure 3

The position of the ball according to the train observer is $x^{\prime}$, and for the observer on the platform will be 


$$
x=x^{\prime}+v t
$$

As you see above, this is exactly Newtonian relativity. But still, we don't agree with Newton with his entire proposal of relativity as clearly explained already. From the above discussion, it is clear to us that the internal energy is constant and the external energy is relative. And since the total energy of an object is the sum of internal and external energies, it implies that the total energy is also relative. To bring some additional prove about the constancy of internal energy, a further discussion take place followed by the same thought experiment that will be carried out to explain the reasons behind the constancy of the speed of light and also prove my second law of energy.

Law 05: The motion of matter or a particle from its internal energy, becomes relative when the velocity is influenced by external energy.

Explanation: The motion of matter or a particle from its internal energy could be influenced by external energy either with increased or decreased velocities of the motion. When the influence of the external energy will be by increased velocity, the motion of the object will be caused within that exact point of time by mere external energy. Because, if the velocity of the external energy was not higher than the motion of external energy, the external energy would have not been able to make that influence on the object. As the velocity of the external energy is higher than the motion of internal energy, the internal energy will not be able to contribute to the motion of the object at that given point in time. As if the light travels through the black hole, the photon then will not be able to travel at its known speed.

But it would travel faster than that because of the gravitational force within the black hole. Under that circumstance, the external energy will be the mere reason for the velocity of the light. Since the external energy took control of the velocity of the object over the internal energy, the motion of that object then will be relative for observers from different frames of reference as discussed earlier in forth law of energy. If the influence of the external energy will be by decreased velocity, it means, the external energy acts in the opposite direction of motion of the object; the internal energy causes the motion of that object is not left to act freely on the object, therefore, the internal energy of motion loses its uniqueness of constancy and it becomes relative at that specific situation. As if the light travels through the water, the one inside the water or on the earth will see the light travels at $3 / 4$ of its constant speed, and the observer from mars for example will find the velocity of the light to be $3 / 4$ of its constant speed plus the velocity of the earth rotates on its axis plus the velocity of the earth in the orbit around the sun.

\section{Law 06: Neither energy can be changed as mass nor mass} as energy.

Explanation: The principle of the existence of matter or a particle is nonexistence, and the principle of ability on an act is inability. To prove the nonexistence or inability of something or someone, we do not need to provide any evidence or proof, because being the principle of something is itself evidence. However, if someone wants to demonstrate that the principle of something is its existence or that of some act is the ability, they must provide proof. Furthermore, if someone submitted substantial evidence on the existence of something or the ability of someone or something on an act, it will become the principle of that particular thing or act. Then, if we want to prove again that the principle of that particular thing is nonexistence or that of the act is inability, we do not need to submit any proof; it is enough to prove that the evidence submitted to claim the existence or the ability is wrong and unacceptable.

For example, there were two people; David and John, and David was killed by someone. For this murder, we cannot accuse John, because according to the principle of acts, John did not kill David. To prove it, there is no need to provide evidence, or else all of us would have to prove our innocence for each crime that occurs anywhere in the world. However, if you want to accuse John of this murder, you have to submit the proof. Once you submit the evidence, the principle of acts will be changed in the case of John from the inability to ability. Thereon, we can say that this incident was caused by John. but if I want to advocate for the innocence of John in this crime, I do not need to submit any evidence exactly for his innocence, but it is enough to prove that the evidence submitted of John's guilt is wrong.

In the same way, according to the principle of matter and particle, there is no such thing as iron, for example. But, if I want to convince you about the existence of iron, I have to provide evidence to prove it. In that case, the principle of matter and particles in the case of iron will be changed from nonexistence to existence.

And based on the principle of acts, you do not have any idea about how to swim. If you want to let us know that you can swim, you have to prove it. Then, the principle of acts will be changed in terms of your swimming capacity from inability to ability.

Just like all these examples, the probability of equating energy and mass is impossible in principle. Therefore, the most famous scientist of the twentieth century, Albert Einstein, had to provide evidence through his well-known equation $E=$ $m c^{2}$ on the possibility of equating energy and mass (Boyer, Einstein, \& Infeld, 1938; Einstein, 1961). From there on, the principle of acts for the equation of energy and mass was rehabilitated from impossibility to possibility and ability. Thus, if I want to claim that there is no probability of equating energy and mass, I do not need to provide any evidence for it, it is enough to prove that Einstein's evidence for equating energy and mass was wrong. I have already done this in the discussion about my first law of energy. So, now we do not have any evidence that proves that the equation of energy and mass is possible. Therefore, this subject is back to its very basic principle, which is inability and impossibility, so we can say that it is not possible to equate energy and mass. Regardless of these arguments, as it was already mentioned in the introduction, Hamdoon's paradoxes of special relativity clearly have proved the inappropriateness of $E=m c^{2}$, and its fundamental ideologies.

Moreover, it is eccentric that we talk about equating energy and mass while it is impossible for a type of energy itself 
(internal energy) to be directly influenced by another type of energy (external energy). Furthermore, equating those two types of energy is impossible.

\section{THE LIGHT}

In our daily lives, light allows us to see and perceive things we would not have seen without it. Unfortunately, there are unanswered questions remaining around light and its velocity, the light of science has not yet fallen upon. We be certain that the speed of light in a vacuum is constant. So, it travels at the same speed irrespective of the distance traveled. Of course, today's scientific research also proves the same. But some unanswered questions also travel as speed as light, beyond the time to date.

a. All the matter and particles we know of, experience changes in their speeds except light, which has a constant velocity in a vacuum. But what is the secret behind its constancy?

b. Is constancy one of the specificities of light, or is there something else that shares the quality of constancy with the speed of light?

These questions have always caused me to think. In the early stages of my research, whenever I tried to dissect these complex questions using the research of expert scientists in this field, I was unsuccessful and failed. Most of the time, I got completely different results. Eventually, I realized that it is impossible to solve these puzzles following the ways and beliefs of existing scientific discoveries. Therefore, I had to approach these questions from a different perspective.

Before we discuss the questions raised above, we will perform a thought experiment:

There was a vehicle traveling at the speed of $28 \mathrm{~m} / \mathrm{s}$, and a person was sitting in the vehicle threw a ball at $6 \mathrm{~m} / \mathrm{s}$ speed. According to the person who threw the ball, its velocity will be $6 \mathrm{~m} / \mathrm{s}$, but for a person observing it from stationary land, the velocity of the ball will be $34 \mathrm{~m} / \mathrm{s}$. With this experiment, we can prove the relativity of external energy as we know it

But suppose the person in the vehicle had switched a light on, instead of throwing the ball? What would the result have been? As we all know, the velocity of the vehicle is never going to make any changes in the speed of the light, it would be the same for the person in the vehicle and the stationary observer. Even if there was a person who had been traveling in another vehicle at $40 \mathrm{~m} / \mathrm{s}$, the velocity would still be $299,792,458 \mathrm{~m} / \mathrm{s}$. Through this experiment along with the postulates of the special theory of relativity, we admit to the constancy of the speed of light (Boyer et al., 1938; Einstein, 1961). Furthermore, we can come to two more conclusions as well.

1. I can prove my second law of energy by this experiment as below:

As discussed in the above experiment, the vehicle was moving at a speed of $28 \mathrm{~m} / \mathrm{s}$. Once the torch was switched on by the person in the vehicle, the light started its journey at $299,792,458 \mathrm{~m} / \mathrm{s}$.

Here, both the vehicle and the light have the same type of energy, which is kinetic energy, even though there were no changes in the velocity of light by the velocity of the vehicle or vice versa. This is because, as I have mentioned for my second law of energy, the kinetic energy of the vehicle does not make any changes in the attributes of the particles of light, which is the photon. Moreover, $299,792,458 \mathrm{~m} / \mathrm{s}$ is the internal energy of the photon. Therefore, no changes were in the speed of light in the above experiment, but if external energy can change internal energy directly without changing the matter or particle, the velocity of the vehicle would have made changes to the velocity of the light. There is perhaps another question that could be asked at this point, which is "assuming that your second law of energy is correct and your proofs are acceptable; how has the speed of the vehicle changed the speed of the ball without changing its attributes?"

The answer to this question is straightforward. Here, the speed of the vehicle is $28 \mathrm{~m} / \mathrm{s}$ and the speed of the ball is $6 \mathrm{~m} / \mathrm{s}$, and both are external energies, and as I have proved already, the external energy is relative. So, it is a common occurrence for external energy to be changed by another external energy. However, 299,792,458 m/s is not the external energy of the photon.

$$
E_{p}=U+T
$$

where $\mathrm{E}_{\mathrm{P}}$ stands for the total energy of a photon, $h \times f=U+$ $T$, where $\mathrm{h}$ stands for Planck's constant, and $f$ represents frequency $U=(h \times f)-T$. Since the external energy of the photon will always be zero, we can say, $U=(h \times f)-0$.

Here, someone may say, "wait a minute. If you say that photon has no external energy when it travels in a vacuum, it is understandable. But how about the external energies, when a photon travels through other media such as water, diamond, and glass, that change the speed of the photon?"

It is an excellent question. I agree that the photon faces some external energies when it travels through such media. But strictly speaking, the external energies the photon faces, under those circumstances, are not the external energies of the photon, because they never act on the photon, but against the photon. And those external energies will be excluded from the equation $E=h f$ already(Planck \& Bohr, 2019). Therefore, the internal energy of the photon will be

$$
U=(h \times f)-0 \quad U=h \times f
$$

And we know that,

$$
\begin{gathered}
c=\lambda \times f \\
f=\frac{c}{\lambda} \quad, \quad U=h \times f
\end{gathered}
$$

or

$$
U=h\left(\frac{c}{\lambda}\right)
$$

Hereby, we can say that the speed of light is the internal energy of a photon. Even though the total energy of a photon is equal to its internal and external energies, only the internal energy of the photon represents its total energy. 
2. Because the speed of light is the internal energy of a photon, it will be the same and constant for all types of observers. This means that the constancy is not the exclusiveness of the velocity of the light. Instead, the light has a constant speed because it is the internal energy of the light and it does not experience any influence of external energy. So, by the above experiment, my third law of energy is also being proved further. Conversely, as I have mentioned already, all internal energies are constant. This means if there is $100 \mathrm{~g}$ of an iron ball at $40{ }^{\circ} \mathrm{C}$, the internal energy of the ball will be the same for all observers, as the speed of light. Even though all types of internal energies are constant, there is a difference between the constancy of the speed of light and the constancy of other internal energies. That is to say, even if the internal energy is constant, we can make changes to it by changing the attribute of the matter or particle, except photons. Since we cannot change the attributes of a photon thus far, we cannot yet change its internal energy. Conversely, if we could change the attributes of the photon, we could change its internal energy. This means that if we can control the attributes of the photon, we can control the speed of light.

\section{CONCLUSIONS}

Different items with the same mass but different attributes or the same items with similar mass and different attributes exhibit different amounts of energy in analogous experiments. This questions our understanding of the relationship between energy and mass. Our close consideration of photon particlesspecifically, the reason for their constant velocity-disproves our belief in the monopoly of the constancy of the speed of light. Furthermore, the photon serves our argument to explain the relationship between energy and mass. Logically it seems as Einstein claims that the value of one buck from the US,

\section{REFERENCES}

Boyer, C., Einstein, A., \& Infeld, L. (1938). The Evolution of Physics: The Growth of Ideas From Early Concepts to Relativity and Quanta. In: Cambridge: Cambridge University Press (2nd edition, 1961).
Canadian, Singapore Jamaican and Liberian dollars are the same since they have a similar unit. Moreover, from our above explanations and understanding, we can draw the following conclusions:

- Energy and mass are not the manifestations of the same thing, but two different manifestations of two entirely different things.

-Not all external energies can make changes to internal energy. -The total energy of matter or a particle cannot be attributed to only the mass and external energy.

-By increasing the velocity, we cannot make any changes to mass, but we can change the external energy of matter or a particle, by which the total energy will be changed.

-By proving the incompleteness of the equation $E=m c^{2}$, we substantiate the impossibility of equating energy and mass.

-The types of energies (internal, external, and total) are not equivalent, but each has differences and uniqueness.

-The speed of the light will become relative when there is an influence of any external energies on its motion.

-The method of our experiments and the results display the relationship between energy and mass from a different and logical perspective that is so related and scientific.

-This paper provides a rudimentary step towards a concrete understanding of energy and mass. If we avoid revalidating our understanding of them, we will be left to stray back and forth until we return to this starting point again and persist with our journey from thereon. Thus, there is an enormous need to reconsider the scientific ideas related to energy and mass; to convert my thought experiments into practical ones that may be abundantly understood; to develop further research based on these fundamental changes. Thus, this paper is a gate that opens the path to revolutionizing science once again.

Einstein, A. (1961). Relativity, the Special and the General Theory: A Popular Exposition. Translated by Robert $W$. Lawson: Crown.

Khan, H. A. J. I. J. o. F. P. S. (2021). The Ultimate Sophistication of Special Theory of Relativity: Relativity. 11(3), 43-49.

Planck, M., \& Bohr, N. (2019). Quantum Theory: Flame Tree Publishing. 